\title{
REVIEW
}

\section{Clinical review: The role of ultrasound in estimating extra-vascular lung water}

\author{
Murali Shyamsundar', Benjamin Attwood², Liza Keating and Andrew P Walden*
}

\begin{abstract}
The estimation of extra-vascular lung water (EVLW) is an essential component in the assessment of critically ill patients. EVLW is independently associated with mortality and its manipulation has been shown to improve outcome. Accurate assessment of lung water is possible with CT and MR imaging but these are impractical for real-time measurement in sick patients and have been superseded by single thermal dilution techniques. While useful, single thermo-dilution requires repeated calibration and is prone to error, suggesting a need for other monitoring methods. Traditionally the lung was not thought amenable to ultrasound examination owing to the high acoustic impedance of air; however, the identification of artefacts in diseased lung has led to increased use of ultrasound as a point of care investigation for both diagnosis and to monitor response to interventions. Following the initial description of B-lines in association with increased lung water, accumulating evidence has shown that they are a useful and responsive measure of the presence and dynamic changes in EVLW. Animal models have confirmed a correlation with lung gravimetry and the utility of B-lines has been demonstrated in many clinical situations and correlated against other established measures of EVLW. With increasing availability and expertise the role of ultrasound in estimating EVLW should be embedded in clinical practice and incorporated into clinical algorithms to aid decision making. This review looks at the evidence for ultrasound as a valid, easy to use, noninvasive point of care investigation to assess EVLW.
\end{abstract}

\section{Introduction}

Extra-vascular lung water (EVLW) is all the fluid within the lungs that is present outside the vascular compartment. Its clinical correlate is pulmonary oedema and it is a function of hydrostatic pressure and pulmonary capillary permeability. In critically ill patients, high levels have been shown to be an independent predictor of outcome [1] and manipulation of the EVLW has also been shown to have a positive effect on outcome [2]. For many years the chest radiograph was used to diagnose changes in EVLW, but this has been shown to have a poor diagnostic accuracy [3]. Other indirect radiological estimates of EVLW, such as CT scanning and MRI, are impractical in critically ill patients, leading to the development of thermal dye techniques to allow more estimation of EVLW at the bedside [4]. Due to the complexity of the dye technique it has largely been superseded by the single thermo-dilution technique, which now constitutes the clinical gold standard of lung water estimation [5]. This technique, however, is invasive and requires regular calibrations, limiting its utility [6].

Traditionally the lung was not felt to be amenable to ultrasound due to its high air content and acoustic impedance; however, the description of common artefacts by Lichtenstein and colleagues [7] and their pathological correlates on CT scanning has led to an expansion in the use of bedside thoracic ultrasound, culminating in evidence-based consensus guidelines [8]. There is now an accumulating body of evidence that lung ultrasound allows a semi-quantitative estimate of lung water and, given its ready availability and ease of use, is a useful point of care tool for this estimation. This paper seeks to lay out the evidence for the use of lung ultrasound in the estimation of EVLW.

\section{EVLW measurement in critically ill patients}

EVLW or EVLW indexed to body weight is an important measure of the state of the lungs in critically ill patients as a high level is independently associated with a worse clinical outcome, including duration of mechanical ventilation, ICU stay and mortality, whether associated with acute lung injury or not $[1,6,9]$. It also appears that manipulation of the EVLW may improve outcome in

\footnotetext{
*Correspondence: andrew.walden@nhs.net

'Intensive Care Unit, Royal Berkshire Hospital, London Road, Reading, Berkshire RG1 5AN, United Kingdom

Full list of author information is available at the end of the article
} 
patients with lung injury [2]. Fluid management aimed at a conservative as opposed to liberal fluid strategy was associated with improved oxygenation index, lung injury score, reduced plateau pressure and increased ventilatorfree days in a pivotal study by the ARDSnet group, further highlighting the importance of limiting EVLW in the critically ill patient population [10]. This was late in the progression of the critical illness and contrary to data on early goal-directed therapy, suggesting that it is not just the amount of fluid but also the timing of fluid that is essential in the management of critically ill patients [11].

Exploring the range of methods available to predict fluid responsiveness in critically ill patients is beyond the remit of this article but the options for monitoring changes in stroke volume and cardiac output are numerous [12-16]. In contrast, the devices available to monitor EVLW in response to changes in fluid status are limited. With a reduction in use of pulmonary artery catheters and given the complexity of dye indicator techniques, the single thermal dilution technique has become the predominant technique for determination of EVLW, derived from the mean transit time of the thermal signal through the lungs.

While this may represent the best compromise between practicability (versus CT/MRI scanning) and portability, it requires central venous and proximal arterial catheters and frequent recalibration for accurate EVLW measurement. There may also be specific methodological issues in critically ill patients where assumptions about chamber ratios and the possibility of shunting and thermal sinks may reduce the accuracy of the results $[17,18]$.

Given these problems and the importance of EVLW in this patient population, a search for more continuous, less invasive measurement is justified. While other modalities such as impedance tomography may be developing a role, only ultrasound gives a direct window on the lung.

Improvements in ultrasound technology in recent years have led to smaller machines and better image quality. As a result of this, ultrasound has moved out of the radiology department to become a point of care test facilitating both rapid diagnosis and dynamic response to interventions. The role of ultrasound is now well established in acute settings like focused assessment with sonography for trauma (FAST) scanning in accident and emergency $[19,20]$, echocardiogram in acute pulmonary embolism [21], thoracic ultrasound for pleural interventions [22] and for vascular access [23]. The evolving evidence base has resulted in the publication of point of care lung ultrasound guidelines [8]

\section{Ultrasound lung comets, lung rockets and the B-line pattern}

The discovery of ultrasound as a rapid, non-invasive and reproducible bedside investigative tool to assess the
EVLW in critically ill patients is worth pursuing as in other similar scenarios, such as vascular access and FAST scanning, it has been shown to be a rapidly acquired skill. The ease of using thoracic ultrasound to measure EVLW was further reinforced by the $99 \%$ feasibility rate achieved in one of the earliest studies conducted by Lichtenstein and colleagues in establishing B-lines as markers of elevated EVLW [7].

The use of ultrasound has expanded further since the significance of the 'comet tail' or B-line artefact was characterised. Lung in its healthy state is poorly penetrated by ultrasound due to the high acoustic impedance of air. In the diseased lung the acoustic impedance may change, allowing better penetration and resolution from ultrasound. In addition, artefacts may develop that can imply disease in the underlying lung. Aerated lung and lung with alveolar/interstitial oedema produce two distinct types of ultrasound artefacts. A repetitive horizontal feature seen in aerated lung called the A-line (Figure 1) is replaced by a vertical narrow based artefact in lungs with alveolar/interstitial oedema called the Bline or comet tail (Figure 2) [7]. The B-line was first demonstrated by Ziskin and colleagues in a patient with abdominal shotgun wound [24]. Areas of differing impedance (for example, the sub-pleural end of a thickened septum), while not visible in ultrasound, results in the beam reflecting indefinitely, leading to a ring down B-line artefact composed of all the microreflections. Lichtenstein and colleagues have shown that B-lines have a high sensitivity and specificity in patients with diffuse alveolar/interstitial oedema [7]. Since then, various studies have correlated the ultrasound evidence of elevated EVLW, that is, B-lines with radiographic and invasive methods of lung water estimation and hydrostatic pressure measurements [25,26].

Nomenclature is important and in this area there are a wide range of terms used - B-lines, lung rockets, ultrasound comet tails. In recognition of this and the potential for confusion, a consensus document has suggested that the term B-line pattern be used [8].

\section{Animal models using ultrasound to estimate EVLW}

The ultimate gold standard for EVLW is gravimetric assessment of the lungs post mortem [27]. Indeed, indicator dye and thermal dilution techniques have been validated in animal models against gravimetric methods showing a bias that could be offset for clinical use [17]. For ultrasound to be accepted as a reasonable alternative to other measures of EVLW it should undergo the same validation in animal models.

This validation was undertaken by Jambrik and colleagues in an oleic acid-induced model of acute lung injury (ALI) in pigs [28]. In this porcine model, oleic acid was administered intravenously in 12 animals to induce 


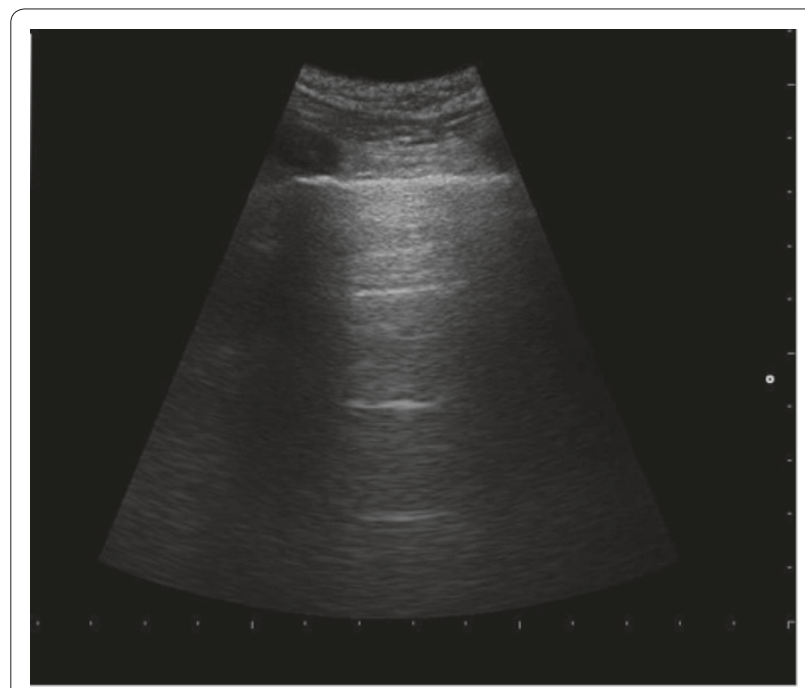

Figure 1. A-lines. Characteristic lines parallel to pleural line as a consequence of ultrasound reverberation artefacts within the pleural space.

ALI while 5 animals where used as controls. Thoracic ultrasound was performed to detect B-lines using a $3.5 \mathrm{~Hz}$ probe at the third and fifth intercostal spaces in the anterior axillary line in each hemithorax. There was a significant increase in B-lines with time after oleic acid administration. There was also a significant co-relation between the number of B-lines at the time of animal sacrifice and wet/dry ratio by gravimetric method.

While the study by Jambrik and colleagues validated Blines to measure EVLW, the more clinically relevant question was if it could detect elevated EVLW before functional impairment becomes apparent. Gargani and colleagues in an oleic acid-induced ALI model in pigs studied the evolution of B-lines in relation to lung compliance and $\mathrm{PaO}_{2} / \mathrm{FiO}_{2}$ (partial pressure of oxygen/ fractional inspired oxygen concentration) ratios [29]. B-lines, compliance and $\mathrm{PaO}_{2} / \mathrm{FiO}_{2}$ ratios were measured every 15 minutes for a total of 90 minutes after the administration of oleic acid in 10 pigs while 5 pigs acted as control. B-lines were measured in six sites in each hemithorax in the third and fifth intercostal spaces using a $7.5 \mathrm{~Hz}$ vascular probe. Mild to moderate ALI was demonstrated in histology with a significant increase in the wet/dry ratio in the lungs of the oleic acid administered pigs when compared to the controls. A significant increase in B-lines was demonstrated in this study at 15 minutes post oleic acid administration with associated reduction in compliance. The increase in B-lines consistently preceded the development of functional impairment signified by a fall in $\mathrm{PaO}_{2} / \mathrm{FiO}_{2}$.

These data suggest that a worsening B-line pattern correlates well with increases in lung water but also that B-lines develop before overt impairment in oxygenation,

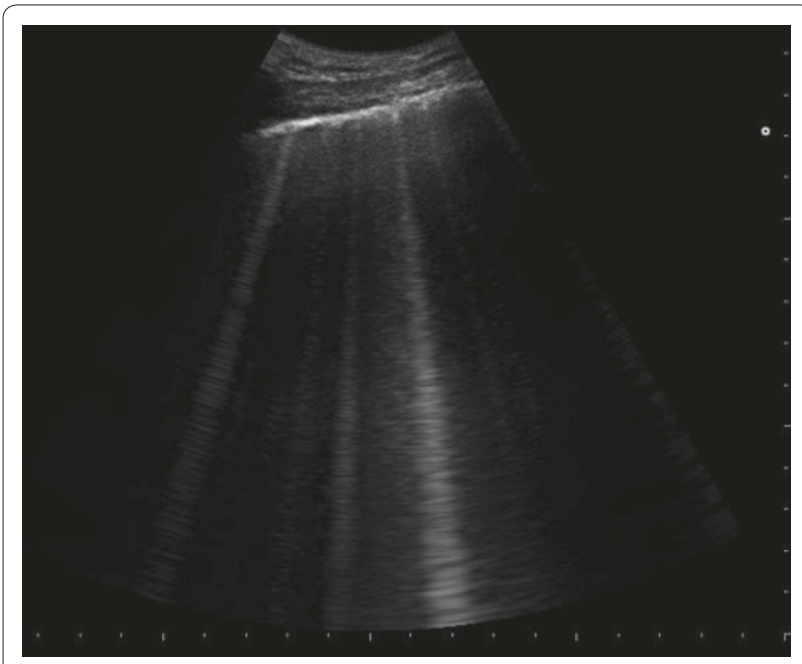

Figure 2. B-lines. Radial lines emanating from the pleural lines due to reverberation artefacts of ultrasound within sub-pleural structures.

suggesting that this may be a useful sign to detect increases in EVLW very early and therefore a potential guide to fluid management.

\section{Validation of B-lines outside critical care}

There is a wealth of data on the validity of B-line pattern correlating with high EVLW outside of critical care. Emergency presentations with shortness of breath, patients with known cardiac disease, fluid overload in the context of chronic haemodialysis and in situations of extreme physiology have all been studied with lung ultrasound. While critically ill patients may have some differences in the underlying pathophysiology, the principles of increased lung water are similar and are a function of both hydrostatic pressures and pulmonary capillary permeability. It is therefore pertinent to examine the data from these settings.

\section{Extreme physiology}

In a study of climbers in Nepal who had a clinical diagnosis of high-altitude pulmonary oedema (HAPE) there was a clear difference in the number of B-lines seen when compared to control subjects without HAPE. Indeed, there was a near linear correlation between the number of B-lines and the oxygen saturations as determined by pulse oximetry [30] and a temporal correlation with improvement in symptoms. Similarly, in another group of 18 climbers in Nepal, the B-line count increased from sea level with increasing altitude and again showed a strong negative correlation with oxygen saturation, making the authors conclude that clinically silent HAPE occurs [31]. Increases in the B-line count have also been demonstrated in elite apnoea divers who are known to suffer with respiratory difficulty after dives due to centralisation 
of blood flow from the peripheries to the pulmonary circulation. The B-line count increased significantly following dives and normalised within 24 hours in a subset [32]. In 31 'Iron man' competitors at sea level there was an increase to what was felt to be a significant level in the B-line count in 23 of them immediately following the race but then decreasing 12 hours after. There were strong correlations between the B-line count and measures of cardiac function, including $\mathrm{N}$-terminal probrain natriuretic peptide (NT pro-BNP) [33].

\section{Emergency department and acute cardiology patients}

There are numerous studies examining the role of B-line estimation in this setting (Table 1). The first demonstration of the use of lung ultrasound to distinguish between chronic obstructive pulmonary disease (COPD) and pulmonary oedema in acutely dyspnoeic patients was demonstrated by Lichtenstein and Meziere in 1998 [34]. Sixty-six consecutive patients with acute dyspnoea, 40 of whom had pulmonary oedema and 26 of whom had exacerbations of COPD, were studied and compared to 80 controls. A B-line pattern was demonstrated in all the patients with pulmonary oedema, only two of the patients with COPD and one control patient, giving a sensitivity of $100 \%$ and specificity of $92 \%$ [34]. Jambrik and colleagues [26] studied 121 patients admitted to a combined cardiology/pulmonology unit, performed lung ultrasound on admission and then compared the B-line count to a blinded assessment of pulmonary oedema from the admission chest X-ray. They found a strong correlation between the B-line count and a validated radiological assessment of pulmonary oedema [26,35]. In a comparative study of 72 patients, 53 with known left ventricular (LV) systolic dysfunction and 19 with normal LV function, patients underwent lung ultrasound and echocardiography before and after exercise. There were strong positive correlations between the B-line count and several measures of cardiac dysfunction, most notably the Echo-derived measure of pulmonary capillary wedge pressure [36,37]. Another analysis was performed on the data from a cohort of 340 patients admitted to a cardiology clinic in Italy [38]. Patients admitted with clinical features consistent with acute pulmonary oedema had a much higher B-line count (50 versus 20 ) and there was also a correlation between the count and New York Heart Association classification, with higher counts being associated with a higher class [38]. The same group examined data on 290 patients admitted with acute dyspnoea or chest pain who were followed up to 16 months and found that B-line count was the strongest predictor of subsequent death or cardiovascular events, even above the ejection fraction [39]. Comparison to other well validated markers of acute LV impairment, such as NT pro-BNP, has also been performed. In 129 patients admitted with acute dyspnoea, a B-line count of greater than 4 was found to have a sensitivity of $81 \%$ and specificity of $85 \%$ and a cutoff of 9 was associated with a sensitivity of $73 \%$ and specificity of $100 \%$ [40]. The area under the receiver operating characteristic curve (AUROC) was 0.893 for lung ultrasound compared to 0.978 for NT pro-BNP [40].

Lichtenstein and Meziere [41] validated a protocol for assessment of acute dyspnoea patients that included a cohort of 64 patients with pulmonary oedema. They used a more simplistic approach of examining the pattern of the anterior lung and defining it as either A-line preponderant or B-line preponderant. When validated against American Heart Association recommendations for the diagnosis of pulmonary oedema together with echo measurements, they found that anterior bilateral B-lines had a sensitivity of $97 \%$ and specificity of $95 \%$ for diagnosing pulmonary oedema [41]. In a similar cohort of 300 consecutive patients admitted to an emergency medical unit, patients underwent lung ultrasound in eight zones (four on either side) and the presence of B-lines in more than two zones was found to have a high sensitivity (85.7\%) and specificity (97.7\%) for diagnosing alveolar-interstitial syndrome [42]. In a group of 81 patients thought clinically likely to have decompensated heart failure, a modified lung ultrasound protocol was performed using 11 intercostal spaces [43]. There were positive correlations between the ultrasound B-line count and the radiological score, clinical score and NT proBNP level. The B-lines resolved following treatment on follow-up scans [43]. More recently, the combination of B-lines and NT pro-BNP differentiated acute decompensated heart failure from both asthma and COPD in a group of 218 emergency department patients [44]. Using the combination gave sensitivity of $100 \%$ and specificity of $100 \%$ [44].

These data support the role of ultrasound in helping to detect increases in EVLW manifested as acute pulmonary oedema.

\section{Haemodialysis patients}

Patients with end stage renal failure on dialysis represent a stable cohort who will have significant fluid shifts around the time of their renal replacement therapy and constitute a useful cohort to study real time changes in fluid status and lung water.

Noble and colleagues found that in 34 of 40 patients with end-stage renal failure examined pre-dialysis, at the mid-point and post-dialysis, there was a significant reduction in B-lines from both pre-dialysis to mid-point and post-dialysis [45]. This has been complemented more recently [46] in 41 patients scanned before and after haemodialysis; there was a strong correlation between the B-line count and the accumulated weight before 
Table 1. Summary of studies assessing the utility of B-line estimation in emergency medicine and cardiology

\begin{tabular}{|c|c|c|c|c|}
\hline Study & Patient group & Ultrasound exam technique & Comparator & Conclusion \\
\hline $\begin{array}{l}\text { Lichtenstein and } \\
\text { Meziere [34] }\end{array}$ & $\begin{array}{l}\text { Acutely dyspnoeic } \\
\text { patients }(n=66)\end{array}$ & $\begin{array}{l}\text { 3.0 MHz cardiac transducer } \\
\text { Anterior - area bound by clavicle to } \\
\text { diaphragm and sternum to AAL } \\
\text { Lateral - area bound by armpit to } \\
\text { diaphragm and AAL to PAL }\end{array}$ & $\begin{array}{l}\text { Clinical, } \\
\text { radiological, Echo }\end{array}$ & $\begin{array}{l}\text { B-line artefact was seen in } 100 \% \text { of patients } \\
\text { with pulmonary oedema and absent in } 92 \% \\
\text { patients with COPD and } 98.75 \% \text { patients with } \\
\text { normal lungs }\end{array}$ \\
\hline $\begin{array}{l}\text { Lichtenstein and } \\
\text { Meziere [41] }\end{array}$ & $\begin{array}{l}\text { Acutely dyspnoeic } \\
\text { patients }(n=260)\end{array}$ & $\begin{array}{l}5 \mathrm{MHz} \text { microconvex transducer } \\
6 \text { sites in each hemithorax divided in } \\
\text { anterior, lateral and posterolateral }\end{array}$ & NA & $\begin{array}{l}\text { Lung ultrasound can reliably distinguish } \\
\text { asthma, COPD, oedema, pulmonary } \\
\text { embolism, pneumothorax and consolidation }\end{array}$ \\
\hline Jambrik et al. [26] & $\begin{array}{l}\text { Cardiology/ } \\
\text { pneumonology }(n=121)\end{array}$ & $\begin{array}{l}2.5 \text { to } 3.5 \mathrm{MHz} \text { cardiac transducer } \\
\text { 2nd to 4th ICS in left hemithorax (to } \\
\text { 5th ICS on the right) at parasternal, } \\
\text { midclavicular, AAL, PAL }\end{array}$ & Chest X-ray & $\begin{array}{l}\text { Strong co-relation between } B \text {-line count and } \\
\text { radiological lung water score }(r=0.78 \text {, } \\
P<0.01)\end{array}$ \\
\hline Agricola et al. [37] & Cardiology $(\mathrm{n}=72)$ & $\begin{array}{l}1.8 \text { to } 3.6 \mathrm{MHz} \text { transducer } \\
\text { 2nd to } 4 \text { th ICS in left hemithorax (to } \\
\text { 5th ICS on the right) at parasternal, } \\
\text { midclavicular, AAL, PAL }\end{array}$ & $\begin{array}{l}\text { Echo (LV systolic } \\
\text { function, PCWP, } \\
\text { PAP) }\end{array}$ & $\begin{array}{l}\text { Positive linear correlations between } \\
\text { baseline B-line count and baseline ejection } \\
\text { fraction, SPAP and estimated PCWP. A similar } \\
\text { co-relation of the difference between } \\
\text { post-exercise and baseline B-line count with } \\
\text { indices of LV systolic and diastolic dysfunction }\end{array}$ \\
\hline Frassi et al. [39] & Cardiology $(n=290)$ & $\begin{array}{l}2.5 \text { to } 3.5 \mathrm{MHz} \text { transducer } \\
\text { 2nd to } 4 \text { th ICS in left hemithorax (to } \\
\text { 5th ICS on the right) at parasternal, } \\
\text { midclavicular, AAL, PAL }\end{array}$ & Echo & $\begin{array}{l}\text { Absence or presence of B-line predicted } \\
\text { event-free survival (70\% versus 19\%, } \\
P<0.0007 \text { ) }\end{array}$ \\
\hline Frassi et al. [38] & $\begin{array}{l}\text { Chest pain/dyspnoea } \\
\text { patients }(n=340)\end{array}$ & $\begin{array}{l}2.5 \text { to } 3.5 \mathrm{MHz} \text { transducer } \\
\text { 2nd to } 4 \text { th ICS in left hemithorax (to } \\
\text { 5th ICS on the right) at parasternal, } \\
\text { midclavicular, AAL, PAL }\end{array}$ & Echo & $\begin{array}{l}\text { B-lines associated with acute cardiac failure, } \\
\text { response to treatment, EDV, LA dimension, } \\
\text { MR, TR }(P<0.0001) \text {. In multivariate analysis } \\
\text { NYHA functional class, EF and diastolic } \\
\text { dysfunction predicted the presence of B-line }\end{array}$ \\
\hline Gargani et al. [40] & $\begin{array}{l}\text { Dyspnoea patients } \\
(\mathrm{n}=149)\end{array}$ & $\begin{array}{l}2.5 \text { to } 3.5 \mathrm{MHz} \text { transducer } \\
\text { 2nd to } 4 \text { th ICS in left hemithorax (to } \\
\text { 5th ICS on the right) at parasternal, } \\
\text { midclavicular, AAL, PAL }\end{array}$ & NT pro-BNP & $\begin{array}{l}\text { B-line co-related to NT pro-BNP ( } r=0.69 \text {, } \\
P<0.0001 \text { ). High accuracy for B-line to } \\
\text { predict aetiology of dyspnoea as of cardiac } \\
\text { origin (AUC 0.893) }\end{array}$ \\
\hline Volpicelli [42] & $\begin{array}{l}\text { Unselected acute } \\
\text { emergency medicine } \\
\text { admissions ( } n=300)\end{array}$ & $\begin{array}{l}3.5 \mathrm{MHz} \text { convex transducer } \\
8 \text { areas in total } \\
\text { Anterior - from the sternum to the } \\
\text { AAL } \\
\text { Lateral - from the AAL to PAL } \\
\text { Each zone was divided into upper } \\
\text { and lower halves }\end{array}$ & Chest X-ray & $\begin{array}{l}\text { Diffuse B lines had sensitivity of } 85.7 \% \text { and a } \\
\text { specificity of } 97.7 \% \text { for diagnosing radiologic } \\
\text { interstitial oedema and a sensitivity of } 85.3 \% \\
\text { and a specificity of } 96.8 \% \text { for diagnosing } \\
\text { a clinical disease with diffuse interstitial } \\
\text { oedema }\end{array}$ \\
\hline Volpicelli et al. [43] & $\begin{array}{l}\text { Acute cardiac failure } \\
(n=81)\end{array}$ & $\begin{array}{l}3.5 \mathrm{MHz} \text { convex transducer } \\
11 \text { areas - } 3 \text { anterior and } 3 \text { lateral on } \\
\text { right side and } 2 \text { anterior and } 3 \text { lateral } \\
\text { on left side }\end{array}$ & $\begin{array}{l}\text { Chest X-ray } \\
\text { NT pro-BNP }\end{array}$ & $\begin{array}{l}\text { Significant resolution of B lines after } \\
\text { treatment }(P<0.001) \text {. Significant co-relation } \\
\text { between B-line and clinical score }(r=0.87 ; \\
P<0.001) \text {, and radiological score }(r=0.62 ; \\
P<0.001) \text { and BNP levels }(r=0.44 ; P<0.05)\end{array}$ \\
\hline Prosen et al. [52] & $\begin{array}{l}\text { Acutely dyspnoeic } \\
\text { patients }(n=218)\end{array}$ & $\begin{array}{l}8 \text { areas in total } \\
\text { Anterior - from the sternum to the } \\
\text { AAL } \\
\text { Lateral - from the AAL to PAL } \\
\text { Each zone was divided into upper } \\
\text { and lower halves }\end{array}$ & $\begin{array}{l}\text { NT pro-BNP } \\
\text { Clinical score }\end{array}$ & $\begin{array}{l}\text { B-line was the strongest predictor of acute } \\
\text { heart failure with 100\% sensitivity, 95\% } \\
\text { specificity, 96\% PPV and 100\% negative NPV } \\
\text { to diagnose cardiac failure. B-line can reliably } \\
\text { exclude pulmonary related dyspnoea in } \\
\text { patients with elevated BNP and a history of } \\
\text { cardiac failure }\end{array}$ \\
\hline Liteplo et al. [53] & $\begin{array}{l}\text { Acutely dyspnoeic } \\
\text { patients }(n=100)\end{array}$ & $\begin{array}{l}\text { 2-5 MHz transducer } \\
8 \text { areas in total } \\
\text { Anterior - from the sternum to the } \\
\text { AAL } \\
\text { Lateral - from the AAL to PAL } \\
\text { Each zone was divided into upper } \\
\text { and lower halves }\end{array}$ & $\begin{array}{l}\text { NT pro-BNP } \\
\text { Clinical review }\end{array}$ & $\begin{array}{l}\text { The presence of a completely positive test } \\
\text { gave an infinite likelihood ratio for diagnosing } \\
\text { congestive cardiac failure and a completely } \\
\text { negative test gave a likelihood ratio of } 0.22 \\
\text { (Cl } 0.06 \text { to 0.8). }\end{array}$ \\
\hline
\end{tabular}

$\mathrm{AAL}$, anterior axillary line; $\mathrm{AUC}$, area under the curve; $\mathrm{Cl}$, confidence interval; $\mathrm{COPD}$, chronic obstructive pulmonary disease; $\mathrm{EDV}$, end diastolic volume; $\mathrm{EF}$, ejection fraction; ICS, intercostal space; LA, left atrium; LV, left ventricle; MR, mitral regurgitation; NA, not available; NPV, negative predictive value; NT pro-BNP, N-terminal probrain natriuretic peptide; NYHA, New York Heart Association; PAL, posterior axillary line; PAP, pulmonary artery pressure; PCWP, pulmonary capillary wedge pressure; PPV, positive predictive value; SPAP, systolic pulmonary artery pressure; TR, tricuspid regurgitation. 
treatment and that lost after dialysis. All ultrasound examinations in this study occurred within a 10 minute period, demonstrating practical, real-time changes.

Mallamaci and colleagues performed chest ultrasound in 75 patients before and after haemodialysis [47]. They grouped patients into three ultrasonographic categories of increasingly severe pulmonary congestion (described as mild, <14 B-lines; moderate, 14 to $30 \mathrm{~B}$-lines; severe, $>30$ B-lines) and found that B-line count significantly reduced after dialysis. They also found significant correlations of pre-dialysis B-lines with early left ventricular filling velocity, early filling to early diastolic mitral annular velocity ratio, pulmonary pressure, and left ventricular end diastolic volume.

These studies give the most convincing data on the cotemporaneous relationship between EVLW, ultrasonographic B-line measurement and hydrostatic pressures, supporting the role of ultrasound as a useful dynamic means of estimating the effects of changes in fluid status on the lung.

\section{Validation of B-lines in critical care}

In a seminal study, Lichtenstein and colleagues [7] enrolled 282 consecutive patients (without pneumothorax) admitted to intensive care and assessed 250 of them for alveolar-interstitial syndrome using conventional chest $\mathrm{X}$-ray against ultrasound examination of the anterolateral chest wall. Alveolar-interstitial syndrome describes a heterogenous group of pathologies associated with increases in lung water (acute respiratory distress syndrome, cardiogenic pulmonary oedema). In 92 patients diagnosed as positive for the syndrome on chest radiograph, they found a pattern consistent with the syndrome in 86 patients using ultrasound (sensitivity of 93.4\%). Absence of positive findings on ultrasound (or confined to the last lateral intercostal space) was noted in 120 of 129 patients with a normal chest X-ray (specificity of 93.0\%). Whilst 29 patients were excluded owing to inconclusive radiography, only 3 patients were excluded because of a non-diagnostic ultrasound exam. The authors concluded that this was the first example in the literature of a relationship between the B-line pattern and alveolar-interstitial syndrome.

Whilst the role of the pulmonary artery catheter (PAC) in the management of critically ill patients has been debated in recent years [48], the measurement of pulmonary artery occlusion pressure (PAOP) remains a reasonable estimate of left heart filling pressures. EVLW is a function of this increase in pressure. A comparison between ultrasound findings and PAOP from PAC has been made in 102 mechanically ventilated patients [49]. Ultrasound provided good correlation, distinguishing between dry interlobular septa (with a predominance of anterior A-lines on ultrasound and a low PAOP value) and alveolar-interstitial syndrome (with a predominance of anterior B-lines on ultrasound and a higher PAOP value). Further to this, they found $\mathrm{B}$-predominance to be present in a wide range of PAOP values. Thus, bedside lung ultrasound could be used to help guide fluid therapy. Ultrasound could provide a useful non-invasive surrogate of PAOP without the potential harm from a PAC.

Similar strong correlation has been shown with the PiCCO device (Pulsion Medical Systems, Munich, Germany) [50]. Agricola and colleagues [50] followed 20 patients undergoing cardiac surgery and studied the assessment of interstitial pulmonary oedema, comparing chest ultrasound against chest radiography, PAC readings and the PiCCO single thermodilution method. They reported a significant positive linear correlation between presence of B-lines, EVLW using the PiCCO single thermodilution method and wedge pressure as measured using the pulmonary artery catheter. There was also high sensitivity (90\%) and specificity (89\%) of the negative test result for detection of EVLW $<500 \mathrm{ml}$. The study did exclude patients with significant lung pathology, reducing the possibility of false-positive results.

\section{Limitations, pitfalls and future directions}

Lung ultrasound is a new technique and has its limitations. It is a user-dependent technique. While previous studies have shown good inter-observer variability, it is important that this is replicated in a broader clinical setting and that training processes and competencies are validated to ensure high quality assurance. It is also important to note that B-lines can be detected in other diseases such as pulmonary fibrosis, although different spacing and artefact patterns may help to differentiate these [51]. B-line artefacts may also be affected by other dynamic changes, such as the application of positive endexpiratory pressure (PEEP) and the respiratory cycle. Above all, it is vital that future research addresses the inconsistency of approaches to quantifying B-lines and attempts to examine the levels of agreement between these measured values against other gold standard techniques such as thermodilution.

\section{Conclusion}

Lung ultrasound is a readily available bedside tool and the correlation of B-lines with EVLW has been demonstrated in animals, extreme environments and a range of clinical settings showing good sensitivity and specificity. The presence or absence of B-lines is a useful tool for ruling in or ruling out pulmonary oedema and alveolar interstitial syndrome in the acute setting and there is evidence of real time matching of B-line quantity with changes in EVLW and total body water. Correlation between PAOP and B-lines in critically ill patients would suggest it is a useful surrogate of left sided filling 
pressures and has a role in guiding fluid filling and resuscitation.

The use of ultrasound in detecting EVLW should be embedded in clinical practice by the development of a protocol to enable its incorporation into a clinical practice algorithm. The protocol should follow the recent guidelines [8] to allow development and implementation of a standardised technique of lung ultrasound.

\section{Abbreviations}

ALI, acute lung injury; COPD, chronic obstructive pulmonary disease; CT, computed tomography; EVLW, extra-vascular lung water; FAST, focused assessment with sonography for trauma; $\mathrm{FiO}_{2}$, fractional inspired oxygen concentration; HAPE, high altitude pulmonary oedema; LV, left ventricle; MRI, magnetic resonance imaging; NT pro-BNP, N-terminal pro-brain natriuretic peptide; PAC, pulmonary artery occlusion pressure; $\mathrm{PaO}_{2}$, partial pressure of oxygen; PAOP, pulmonary artery occlusion pressure.

\section{Author details}

${ }^{1}$ Intensive Care Unit, Royal Berkshire Hospital, London Road, Reading, Berkshire, RG1 5AN, United Kingdom. ${ }^{2}$ Intensive Care Unit, John Radcliffe Hospital, Oxford, United Kingdom.

\section{Competing interests}

The authors declare that they have no competing interests.

\section{Published: 13 September 2013}

\section{References}

1. Sakka S, Klein M, Reinhart K, Meier-Hellmann A: Prognostic value of extravascular lung water in critically ill patients. Chest 2002, 122:2080-2086.

2. Mitchell JP, Schuller D, Calandrino FS, Schuster DP: Improved outcome based on fluid management in critically ill patients requiring pulmonary artery catheterization. Am Rev Respir Dis 1992, 145:990-998.

3. Halperin BD FT, Mihm FG, Chiles C, Guthaner DF, Blank NE: Evaluation of the portable chest roentgenogram for quantitating extravascular lung water in critically ill adults. Chest 1985, 88:649-652.

4. Lewis FR, Elings VB, Hill SL, Christensen JM: The measurement of extravascular lung water by thermal-green dye indicator dilution. Ann NY Acad Sci 1982, 384:394-410.

5. Sakka SG, Rühl CC, Pfeiffer UJ, Beale R, McLuckie A, Reinhart K, MeierHellmann A: Assessment of cardiac preload and extravascular lung water by single transpulmonary thermodilution. Intensive Care Med 2000, 26:180-187.

6. Craig TR DM, Shyamsundar M, McDowell C, McLaughlin B, Elborn JS, McAuley DF: Extravascular lung water indexed to predicted body weight is a novel predictor of intensive care unit mortality in patients with acute lung injury. Crit Care Med 2010, 38:114-120

7. Lichtenstein D, Meziere G, Biderman P, Gepner A, Barre O: The comet-tail artifact. An ultrasound sign of alveolar-interstitial syndrome. Am J Respir Crit Care Med 1997, 156:1640-1646.

8. Volpicelli G, Elbarbary M, Blaivas M, Lichtenstein DA, Mathis G, Kirkpatrick AW, Melniker L, Gargani L, Noble VE, Via G, Dean A, Tsung JW, Soldati G, Copetti R, Bouhemad B, Reissig A, Agricola E, Rouby JJ, Arbelot C, Liteplo A, Sargsyan A, Silva F, Hoppmann R, Breitkreutz R, Seibel A, Neri L, Storti E, Petrovic T; International Liaison Committee on Lung Ultrasound (ILC-LUS) for International Consensus Conference on Lung Ultrasound (ICC-LUS): International evidence-based recommendations for point-of-care lung ultrasound. Intensive Care Med 2012, 38:577-591.

9. Phillips CR CM, Smith SM: Extravascular lung water in sepsis-associated acute respiratory distress syndrome: indexing with predicted body weight improves correlation with severity of illness and survival. Crit Care Med 2008, 36:69-73.

10. The National Heart L, and Blood Institute Acute Respiratory Distress Syndrome (ARDS) Clinical Trials Network: Comparison of two fluidmanagement strategies in acute lung injury. N Engl J Med 2006, 354:2564-2575.

11. Rivers E, Nguyen B, Havstad S, Ressler J, Muzzin A, Knoblich B, Peterson E, Tomlanovich M; Early Goal-Directed Therapy Collaborative Group: Early goal-directed therapy in the treatment of severe sepsis and septic shock. NEngl J Med 2001, 345:1368-1377.

12. Tavernier $B$, Makhotine $O$, Lebuffe $G$ : Systolic pressure variation as a guide to fluid therapy in patients with sepsisinduced hypotension.

Anesthesiology 1998, 89:1313-1321.

13. Michard F, Boussat S, Chemla D, Anguel N, Mercat A, Lecarpentier Y, Richard C, Pinsky MR, Teboul JL: Relation between respiratory changes in arterial pulse pressure and fluid responsiveness in septic patients with acute circulatory failure. Am J Respir Crit Care Med 2000, 162:134-138.

14. Magder S: How to use central venous pressure measurements. Curr Opin Crit Care 2005, 11:264-270.

15. Michard FTJ: Predicting fluid responsiveness in ICU patients: a critical analysis of the evidence. Chest 2002, 121:2000-2008.

16. Belda FJ, Aguilar G, Teboul JL, Pestaña D, Redondo FJ, Malbrain M, Luis JC, Ramasco F, Umgelter A, Wendon J, Kirov M, Fernández-Mondéjar E; PICS Investigators Group: Complications related to less-invasive haemodynamic monitoring. Br J Anaesth 2011, 106:482-486.

17. Effros RM, Pornsuriyasak P, Porszasz J, Casaburi R: Indicator dilution measurements of extravascular lung water: basic assumptions and observations. Am J Physiol Lung Cell Mol Physiol 2008, 294:L1023-1031.

18. Brown LM, Liu KD, Matthay MA: Measurement of extravascular lung water using the single indicator method in patients: research and potential clinical value. Am J Physiol Lung Cell Mol Physiol 2009, 297:L547-558.

19. Ollerton JE, Sugrue M, Balogh Z, D'Amours SK, Giles A, Wyllie P: Prospective study to evaluate the influence of FAST on trauma patient management. J Trauma 2006, 60:785-791.

20. Melniker LA, Leibner E, McKenney MG, Lopez P, Briggs WM, Mancuso CA: Randomized controlled clinical trial of point-of-care, limited ultrasonography for trauma in the emergency department: the first sonography outcomes assessment program trial. Ann Emerg Med 2006 48:227-235.

21. Lodato JA, Ward RP, Lang RM: Echocardiographic predictors of pulmonary embolism in patients referred for helical CT. Echocardiography 2008, 25:584-590.

22. Diacon $\mathrm{AH}$, Brutsche MH, Soler M: Accuracy of pleural puncture sites. A prospective comparison of clinical examination with ultrasound. Chest 2003, 123:436-441.

23. Kumar A, Chuan A: Ultrasound guided vascular access: efficacy and safety. Best Pract Res Clin Anaesthesiol 2009 23:299-311.

24. Ziskin MC, Thickman DI, Goldenberg NJ, Lapayowker MS, Becker JM: The comet tail artifact. J Ultrasound Med 1982, 1:1-7

25. Picano E, Frassi F, Agricola E, Gligorova S, Gargani L, Mottola G: Ultrasound lung comets: a clinically useful sign of extravascular lung water. J Am Soc Echocardiogr 2006, 19:356-363.

26. Jambrik Z, Monti S, Coppola V, Agricola E, Mottola G, Miniati M, Picano E: Usefulness of ultrasound lung comets as a nonradiologic sign of extravascular lung water. Am J Cardio/ 2004, 93:1265-1270.

27. Pearce ML YJ, Beazell J: Measurement of pulmonary edema. Circ Res 1965 $16: 482-488$.

28. Jambrik Z, Gargani L, Adamicza A, Kaszaki J, Varga A, Forster T, Boros M, Picano $\mathrm{E}$ : B-lines quantify the lung water content: a lung ultrasound versus lung gravimetry study in acute lung injury. Ultrasound Med Biol 2010, 36:2004-2010

29. Gargani L, Lionetti V, Di Cristofano C, Bevilacqua G, Recchia FA, Picano E: Early detection of acute lung injury uncoupled to hypoxemia in pigs using ultrasound lung comets. Crit Care Med 2007, 35:2769-2774.

30. Fagenholz PJ, Gutman JA, Murray AF, Noble VE, Thomas SH, Harris NS: Chest ultrasonography for the diagnosis and monitoring of high-altitude pulmonary edema. Chest 2007, 131:1013-1018.

31. Pratali L, Cavana M, Sicari R, Picano E: Frequent subclinical high-altitude pulmonary edema detected by chest sonography as ultrasound lung comets in recreational climbers. Crit Care Med 2010, 38:1818-1823.

32. Frassi F, Pingitore A, Cialoni D, Picano E: Chest sonography detects lung water accumulation in healthy elite apnea divers. J Am Soc Echocardiogr 2008, 21:1150-1155.

33. Pingitore A, Garbella E, Piaggi P, Menicucci D, Frassi F, Lionetti V, Piarulli A, Catapano G, Lubrano V, Passera M, Di Bella G, Castagnini C, Pellegrini S, Metelli MR, Bedini R, Gemignani A, L'Abbate A: Early subclinical increase in pulmonary water content in athletes performing sustained heavy exercise at sea level: ultrasound lung comet-tail evidence. Am J Physiol Heart Circ Physiol 2011, 301:H2161-2167. 
34. Lichtenstein $\mathrm{D}$, Meziere $\mathrm{G}$ : A lung ultrasound sign allowing bedside distinction between pulmonary edema and COPD: the comet-tail artifact. Intensive Care Med 1998, 24:1331-1334

35. Giuntini C, Pistolesi M, Miniati M, Fazio F: Theoretical and practical considerations of measuring extravascular lung water. J Thorac Imaging 1988, 3:36-43.

36. Nagueh SF, Middleton KJ, Kopelen HA, Zoghbi WA, Quinones MA: Doppler tissue imaging: a noninvasive technique for evaluation of left ventricular relaxation and estimation of filling pressures. J Am Coll Cardiol 1997, 30:1527-1533.

37. Agricola E, Picano E, Oppizzi M, Pisani M, Meris A, Fragasso G, Margonato A: Assessment of stress-induced pulmonary interstitial edema by chest ultrasound during exercise echocardiography and its correlation with left ventricular function. J Am Soc Echocardiogr 2006, 19:457-463.

38. Frassi F, Gargani L, Gligorova S, Ciampi Q, Mottola G, Picano E: Clinical and echocardiographic determinants of ultrasound lung comets. Eur J Echocardiogr 2007, 8:474-479.

39. Frassi F, Gargani L, Tesorio P, Raciti M, Mottola G, Picano E: Prognostic value of extravascular lung water assessed with ultrasound lung comets by chest sonography in patients with dyspnea and/or chest pain. J Card Fail 2007, 13:830-835

40. Gargani L, Frassi F, Soldati G, Tesorio P, Gheorghiade M, Picano E: Ultrasound lung comets for the differential diagnosis of acute cardiogenic dyspnoea: a comparison with natriuretic peptides. Eur I Heart Fail 2008, 10:70-77.

41. Lichtenstein DA, Meziere GA: Relevance of lung ultrasound in the diagnosis of acute respiratory failure: the BLUE protocol. Chest 2008, 134:117-125

42. Volpicelli G, Mussa A, Garofalo G, Cardinale L, Casoli G, Perotto F, Fava C, Frascisco M: Bedside lung ultrasound in the assessment of alveolarinterstitial syndrome. Am J Emerg Med 2006, 24:689-696.

43. Volpicelli G, Caramello V, Cardinale L, Mussa A, Bar F, Frascisco MF: Bedside ultrasound of the lung for the monitoring of acute decompensated heart failure. Am J Emerg Med 2008, 26:585-591.

44. Prosen G, Klemen P, Strnad M, Grmec S: Combination of lung ultrasound (a comet-tail sign) and $\mathrm{N}$-terminal pro-brain natriuretic peptide in differentiating acute heart failure from chronic obstructive pulmonary disease and asthma as cause of acute dyspnea in prehospital emergency setting. Crit Care 2011, 15:R114.

45. Noble VE, Murray AF, Capp R, Sylvia-Reardon MH, Steele DJ, Liteplo A: Ultrasound assessment for extravascular lung water in patients undergoing hemodialysis. Time course for resolution. Chest 2009, 135:1433-1439.

46. Trezzi M, Torzillo D, Ceriani E, Costantino G, Caruso S, Damavandi PT, Genderini A, Cicardi M, Montano N, Cogliati C: Lung ultrasonography for the assessment of rapid extravascular water variation: evidence from hemodialysis patients. Intern Emerg Med 2011 [Epub ahead of print].

47. Mallamaci F, Benedetto FA, Tripepi R, Rastelli S, Castellino P, Tripepi G, Picano E, Zoccali C: Detection of pulmonary congestion by chest ultrasound in dialysis patients. JACC Cardiovasc Imaging 2010, 3:586-594.

48. Rajaram SS, Desai NK, Kalra A, Gajera M, Cavanaugh SK, Brampton W, Young D, Harvey S, Rowan K: Pulmonary artery catheters for adult patients in intensive care. Cochrane Database Syst Rev 2013, 2:CD003408.

49. Lichtenstein DA, Meziere GA, Lagoueyte JF, Biderman P, Goldstein I, Gepner A: A-lines and B-lines: lung ultrasound as a bedside tool for predicting pulmonary artery occlusion pressure in the critically ill. Chest 2009, 136:1014-1020.

50. Agricola E, Bove T, Oppizzi M, Marino G, Zangrillo A, Margonato A, Picano E: "Ultrasound comet-tail images": a marker of pulmonary edema: a comparative study with wedge pressure and extravascular lung water. Chest 2005, 127:1690-1695.

51. Copetti R, Soldati G, Copetti P: Chest sonography: a useful tool to differentiate acute cardiogenic pulmonary edema from acute respiratory distress syndrome. Cardiovasc Ultrasound 2008, 6:16.

52. Prosen G, Klemen P, Strnad M, Grmec S: Combination of lung ultrasound (a comet-tail sign) and $\mathrm{N}$-terminal pro-brain natriuretic peptide in differentiating acute heart failure from chronic obstructive pulmonary disease and asthma as cause of acute dyspnea in prehospital emergency setting. Crit Care 2011, 15:R114.

53. Liteplo AS, Marill KA, Villen T, Miller RM, Murray AF, Croft PE, Capp R, Noble VE: Emergency thoracic ultrasound in the differentiation of the etiology of shortness of breath (ETUDES): sonographic B-lines and N-terminal probrain-type natriuretic peptide in diagnosing congestive heart failure. Acad Emerg Med 2009, 16:201-210.

doi:10.1186/cc12710

Cite this article as: Shyamsundar M, et al: Clinical review: The role of ultrasound in estimating extra-vascular lung water. Critical Care 2013, 17:237. 